\title{
Synthetic histone code
}

\section{Wolfgang Fischle ${ }^{1}$, Henning D Mootz ${ }^{2}$ and Dirk Schwarzer ${ }^{3}$}

\begin{abstract}
Chromatin is the universal template of genetic information in all eukaryotic cells. This complex of DNA and histone proteins not only packages and organizes genomes but also regulates gene expression. A multitude of posttranslational histone modifications and their combinations are thought to constitute a code for directing distinct structural and functional states of chromatin. Methods of protein chemistry, including protein semisynthesis, amber suppression technology, and cysteine bioconjugation, have enabled the generation of so-called designer chromatin containing histones in defined and homogeneous modification states. Several of these approaches have matured from proof-of-concept studies into efficient tools and technologies for studying the biochemistry of chromatin regulation and for interrogating the histone code. We summarize pioneering experiments and recent developments in this exciting field of chemical biology.
\end{abstract}

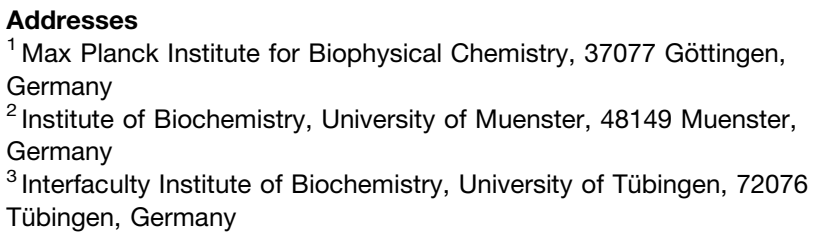

Corresponding authors: Fischle, Wolfgang (wfischl@gwdg.de), Mootz, Henning D (henning.mootz@unimuenster.de) and Schwarzer, Dirk (dirk.schwarzer@uni-tuebingen.de)

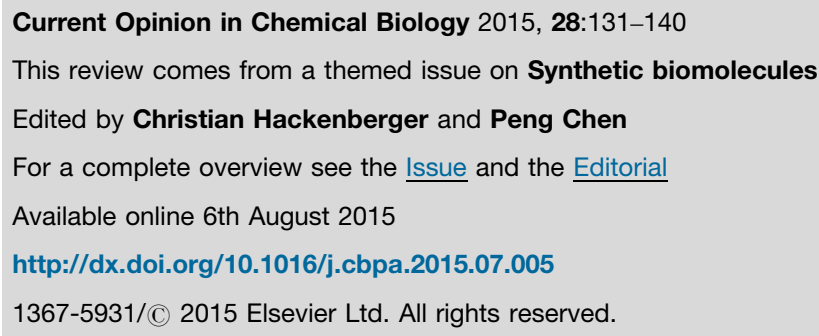

\section{Chromatin and posttranslational histone modifications}

The physiological template of genetic information in all eukaryotic cells is chromatin, the complex of DNA and histone proteins. As signaling platform, chromatin integrates a variety of internal and external cellular inputs. These direct distinct local and global structural and functional states of chromatin, thereby controlling gene expression [1].

In the repeating unit of chromatin, the nucleosome, DNA is wrapped around an octamer of histone proteins (two copies each of the core histones $\mathrm{H} 2 \mathrm{~A}, \mathrm{H} 2 \mathrm{~B}, \mathrm{H} 3$, and $\mathrm{H} 4$ and one copy of linker histone H1, Figure 1a) [1]. By various levels of folding, the originating primary chromatin fiber can be organized into various structural arrangements, including highly condensed mitotic chromosomes (Figure 1b). While the basic architecture of nucleosomes is the same throughout the genome, posttranslational modifications (PTMs) of histones are central means of increasing the biochemical divergence that regulates chromatin structure and function [2,3]. Histone PTMs are structurally diverse and include methylation, acetylation and ubiquitinylation of lysine, as well as phosphorylation of serine and threonine residues (Figure 1c). More than 150 histone modification sites have been identified in different experimental systems. Major sites of modification cluster within the unstructured regions of the Nterminal histone tails that vary between 10 and 35 amino acids in length. These are protruding out from the nucleosome core (Figure 1a).

Research over the past years has focused on defining the distribution, biochemistry and cellular function of individual and combinations of histone marks. A 'histone code' hypothesis has been put forward that defines chromatin as dynamic programming platform, which integrates internal and external cellular signals [2,3]. Histone modifying enzymes are considered the writers of the histone code. Chromatin factors possessing PTM-binding domains serve as histone code readers and execute regulatory functions upon recruitment. Finally, dedicated PTM-removing enzymes are the erasers of histone PTMs [4-7].

Deciphering the complex cross-talk between histone PTMs, chromatin factors and gene regulation is a major research challenge [8]. While the accessibility of 'designer chromatin' composed of histones with defined and homogeneous modification states has been a central bottleneck in chromatin research, novel chemical approaches are providing powerful tools enabling important discoveries of the rules of the histone code [4-7,9-12].

\section{Tools for designer chromatin}

Chromatin research has benefitted strongly from peptide chemistry. Especially, the availability of building blocks of modified amino acids has enabled the synthesis of histone peptides with defined modification patterns. Such histone-derived peptides have been instrumental for many key discoveries on the readers, writers and eraser of histone PTMs. However, histone-peptides can only recapitulate a minor fraction of the complex chromatin structure (Figure 1). Consequently, major efforts have been untertaken to site-specifically introduce 


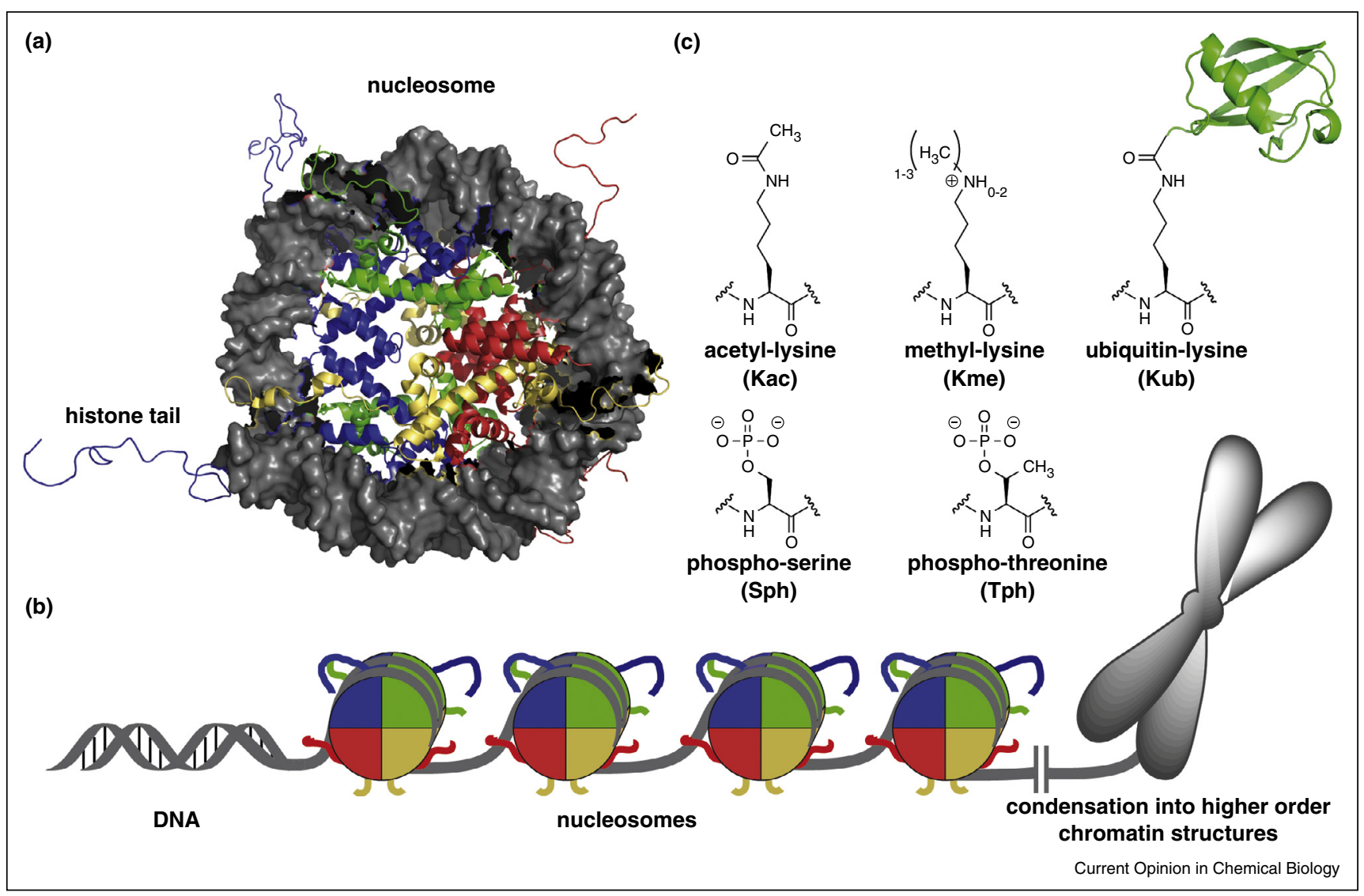

Histones, nucleosomes and chromatin. (a) Structure of the nucleosome core particle. Histones are color-coded as follows: H2A: yellow, H2B: red, H3: blue, H4: green. The illustration was generated from pdb file: 1KX5. (b) Packaging of DNA into nucleosomes and chromosomes. Intermediate packaging stages are not illustrated. (c) Selected posttranslational modifications of histones including acetylation, methylation and ubiquitination of lysine as well as phosphorylation of serine and threonine residues.

modification marks into full-length histones, nucleosomes, arrays of nucleosomes and even into chromatin of living cells. These approaches draw from the technological repertoire of modern protein chemistry [4-7].

\section{Selective modification of cysteine residues}

The most widely used strategy for generating modified histones is the selective conversion of cysteine residues into mimics of modified amino acids. The chemical properties of the cysteine thiol group are unique among the proteinogenic amino acids. Selective alkylation of this soft nucleophile has been used in traditional biochemistry and this approach has recently experienced a renaissance for site-specific introduction of mimics of P'TMs. Since natural cysteine residues are absent in core histones with exception of a single residue in $\mathrm{H} 3$, this method is particularly attractive for chromatin chemistry. In a pioneering report site-directed mutagenesis was used to introduce cysteine at sites of methylated lysine residues in otherwise Cys-free mutant histone $\mathrm{H} 3$ as well as histone $\mathrm{H} 4$ [13]. Upon treatment with 2-chloro-ethyl-methylammonium or bromo-ethylmethylammonium compounds (1) the respective methyl-thialysine (2) residues were generated in high yields (Figure 2a). Downstream experiments showed that these so-called methyl-lysine analogs (MLAs) mimicked properties of methylated lysine in peptide and nucleosomal context by recruiting chromatin readers.

In a similar fashion methyl-arginine analogs (4) were generated [14]. In this case $\alpha, \beta$-unsaturated amidines (3) served as alkylation agents. Extending this approach to acetyl-thialysine (6) turned out much more challenging [15]. The treatment of cysteine with 2-bromoethyl-acetylamine $(5)$ or with more reactive $\mathrm{N}$-acetyl-aziridine, were either poor yielding or led to undesired side-products. Alkylation with methylthiocarbonyl-aziridine (7) converted cysteine residues into methylthiocarbonyl-thialysine (8), which mimicked some aspects of acetylated lysine [15]. Efficient formation of acetyl-thiaLys (6) was finally achieved with N-vinyl-acetamidine (9), however not by direct conjugation but via the radical thiol-ene 


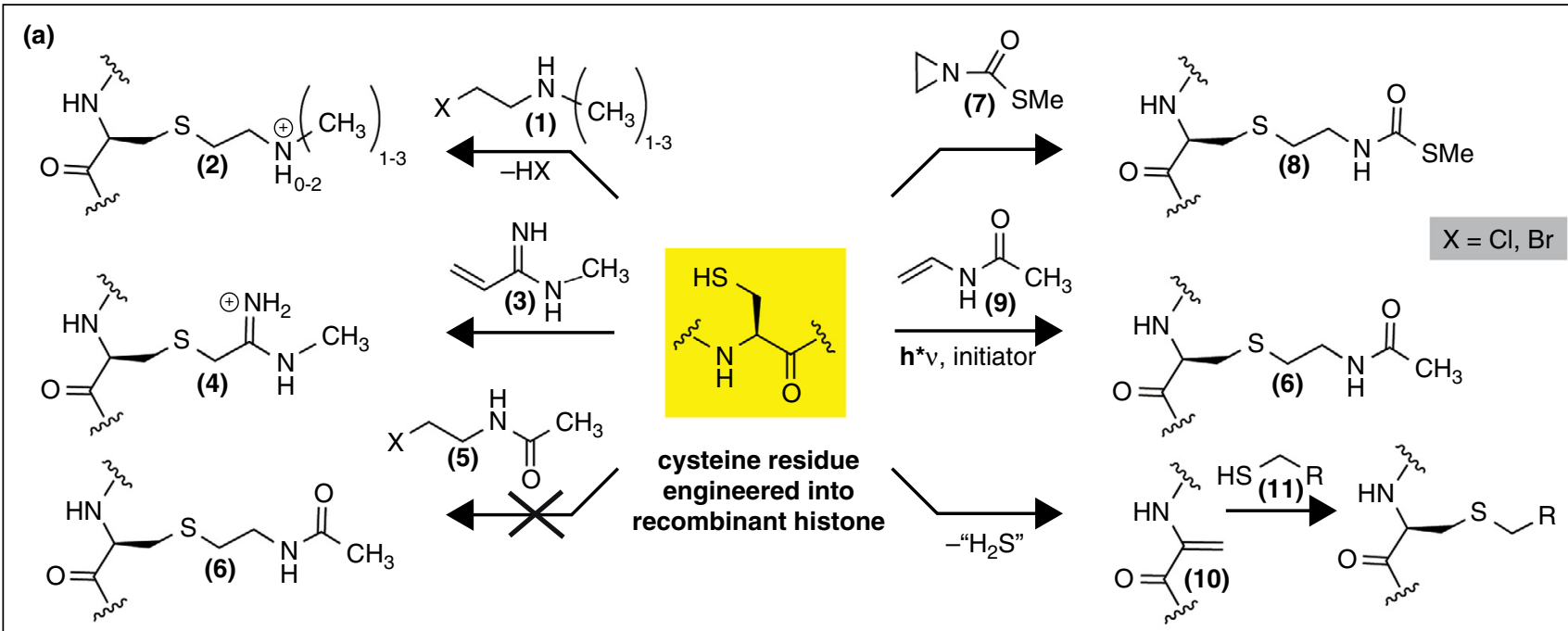

(b)<smiles>CC(=O)NCCCC[C@H](N)C(=O)[O-]</smiles>

Ne-acetyl-lysine

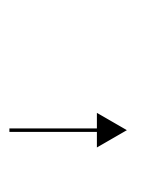

recom recombinant histone containing acetyl-lysine

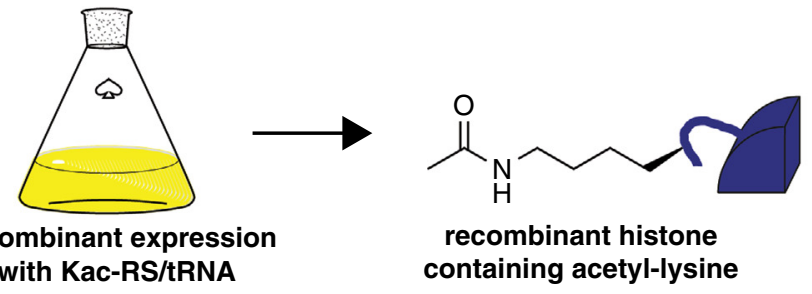

(c)

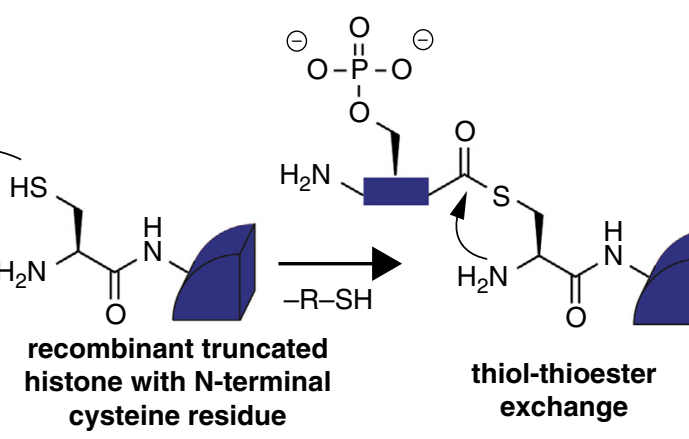

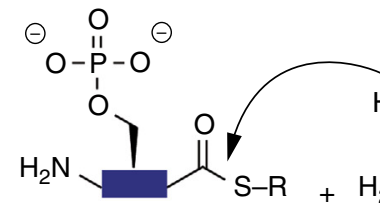

synthetic modified $\mathrm{N}$-terminal tail peptide thioester recombinant truncated cysteine residue exchange

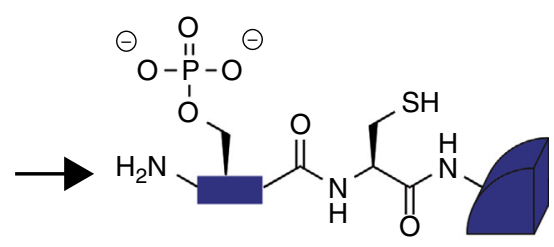

semisynthetic histone with modified $\mathrm{N}$-terminal tail

(d)

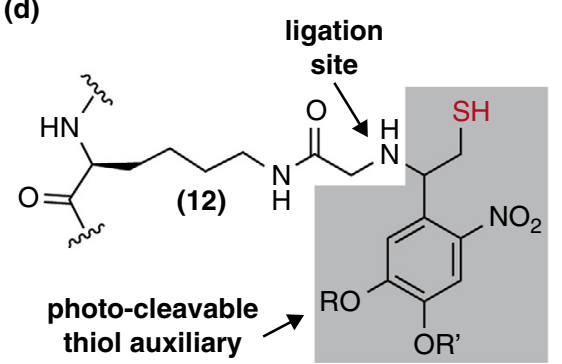

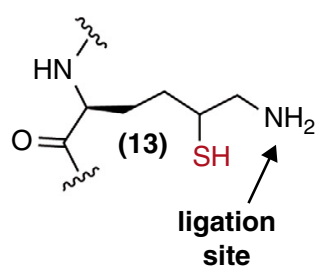

$12+$ Ub-thioester (NCL)

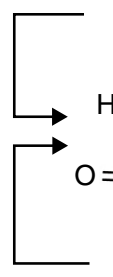

cleavage of auxiliary<smiles>[134IH]</smiles><smiles>CCN</smiles>

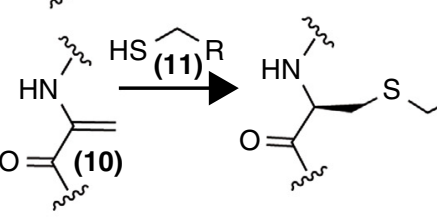

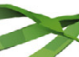

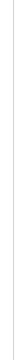


reaction [16]. A promising alternative to the direct modification is the selective elimination of the cysteine thiol resulting in dehydroalanine (10), which can serve as acceptor for thiol-containing nucleophiles (11) in Michael reactions [17].

Despite the applicability of this approach it needs to be emphasized that thialysine and its derivatives are isosteric mimics but differ from lysine with respect to the $\mathrm{p} K_{\mathrm{a}}$ of side-chain amine as well as the molecular geometry at the thioether bond. Consequently, quantitative studies on methyllysine reader domains and lysine demethylases have uncovered significant differences between natural substrates and mimics in terms of binding and catalytic properties [18-20].

Site-specific histone ubiquitylation by disulfide formation represents a further approach useful for coupling small proteins to histones. Via an isopeptide bond the C-terminus of the 76 aa ubiquitin polypeptide is naturally linked to the $\varepsilon$-amino group of lysine. While the formed disulfide moieties differ significantly from the native lysine modification, this method is highly efficient in terms of synthesis. The disulfide-bound ubiquitin moieties were shown to mimic the natural modification state well [21]. Disulfides are not stable under reducing conditions, which can complicate reaction procedures. Nevertheless, the simple erasure of this modification mark by reducing agents might be useful in specific experimental settings.

\section{Amber suppression technology}

A powerful alternative approach to chemical side chain modification is direct genetic encoding of modified amino acids by the amber suppression technology (Figure 2b). An 'empty' stop codon (commonly the amber codon) is read during ribosomal translation by an extra tRNA that is co-expressed together with its cognate aminoacyl-tRNA synthetase (RS). The latter one is obtained by genetic engineering and protein evolution to recognize the desired amino acid [22]. The most widely used RS for chromatin-relevant modifications, PylRS, is responsible for esterification of the 22nd proteinogenic amino acid pyrrolysine to its cognate tRNA. It displays relaxed substrate selectivity and is able to process selected $\mathrm{N} \varepsilon$ acyl lysine derivatives without engineering [23]. The repertoire of encoded non-natural amino acids has expanded vastly in recent years. Well-known examples are fluorescent amino acids, photo-crosslinkers and amino acids that carry special functional groups, which are otherwise absent from proteins [24]. The aldehydes, alkynes, azides or additional thiols enable downstream modification with probes or modifications including ubiquitin and SUMO [25-28]. Also, encoded amino acids that carry PTMs, such as acetyllysine, various acyllysine (propionyllysine, butyryllysine and crotonyllysine) derivatives and a protected mono-methyllysine have been already successfully introduced into histones [23,29-32]. A further important modified amino acid that was recently encoded is phospho-serine; in this case by hijacking an O-phosphoserylRS involved in cysteinyl-tRNA synthesis of archaea $\left[33,34^{\circ}\right]$.

\section{Protein semisynthesis}

Protein semisynthesis is a collective term for chemoselective ligation strategies of synthetic peptides and recombinant proteins, which allow reconstitution of whole proteins. Chemical synthesis of one of these peptide fragments enables the introduction of structural variations with virtually no restrictions regarding their nature and number [35,36]. Native chemical ligation (NCL) and the related expressed protein ligation (EPL) are based on the selective ligation of thioesters with peptides or proteins possessing an N-terminal Cys residue (Figure 2c) $[35,36]$.

In recent years NCL-based ligation approaches were applied to all of the four core histones. This includes strategies for introducing different PTMs in the tails of histones $\mathrm{H} 3$ and $\mathrm{H} 4$ as well as elaborate ligation schemes for site-specific coupling of ubiquitin at the C-terminus of histone H2B [37-43]. In the latter, a photo-cleavable thiol auxiliary (12) mediated NCL between an ubiquitinthioester and the respective lysine side chain in a peptide derived from H2B (Figure 2d) [41]. In this case the Cterminal glycine residue of ubiquitin was linked to the lysine residue of the $\mathrm{H} 2 \mathrm{~B}$ peptide. The corresponding ubiquitin thioester was truncated by this C-terminal glycine, thereby placing the ligation site within the ubiquitin protein (Figure 2d). Subsequent cleavage of the auxiliary and uncaging of an $\mathrm{N}$-terminal cysteine of the H2B peptide by UV light established the native isopeptide connection with ubiquitin and enabled a second NCL reaction with a recombinant $\mathrm{N}$-terminal histone fragment containing a C-terminal thioester.

An alternative approach for chemically attaching the ubiquitin PTM to a lysine side chain employs the nonnatural amino acid $\delta$-mercaptolysine (13) $\left[44^{\circ}\right]$. The additional thiol in the side chain enables NCL with the NE amino group of this lysine derivative, which also establishes an isopeptide bond with ubiquitin thioesters (Figure 2d) [43]. A potential problem of NCL-like ligation schemes arises from the thiol-containing residue that mediates the reaction and remains in the ligation products as mutation. However, efficient and specific desulfurization strategies have been developed for removing the thiol moieties after the ligation [45]. Finally, it should be noted that NCL-like strategies have been applied for the total chemical synthesis of histones, which provides chemical control over each residue of the synthetic proteins $[43,46,47]$.

In contrast to the chemical ligation approaches to reconstitute the peptide backbone of a protein from two fragments, sortase-mediated ligation and protein 
trans-splicing by split inteins represent chemo-enzymatic technologies [48-51]. The bacterial transpeptidase sortase A recognizes a highly conserved so-called sorting motif consisting of the amino acid sequence LPxTG (X: any amino acid) in one of the ligation partners. The enzyme cleaves the motif at the threonine residue under concomitant formation of a sortase-bound thioester, which is ligated to a peptide or protein possessing an N-terminal Gly residue [49,51]. Histone H3 contains an amino acid sequence, which differs in a single residue (APxTG) from the sorting motif and this sequence is located at the interface between tail and the globular fold. Protein evolution approaches have been put forth to generate a sortase mutant that ligates the $\mathrm{H} 3$ sequence rather than the native sorting motif, thereby enabling traceless semisynthesis of this histone [52].

Protein trans-splicing mediates the ligation between two polypeptides that bear at their $\mathrm{C}$-termini and $\mathrm{N}$-termini the N-terminal and C-terminal halves of a split intein (peptide ${ }^{\mathrm{N}}$-intein $^{\mathrm{N}}$ and intein ${ }^{\mathrm{C}}$-peptide ${ }^{\mathrm{C}}$ ) $[48,50]$. Since the intein parts remove themselves during the reaction and leave behind only a single Cys, Ser or Thr residue at the splice junction, the reaction can be designed in a traceless fashion. The other key advantage of split inteins is the inherent affinity between the two halves, thereby enabling protein reconstitution at low concentrations (down to nanomolar). Combined with the selectivity and bioorthogonality of the reaction, split inteins are highly attractive for applications in a cellular context $[53,54]$.

\section{Designer chromatin in biochemistry and biology}

The availability of histones of defined modification status has enabled a level of in vitro biochemical investigation of chromatin structure and function that would not have been accessible by conventional approaches. Classically, chromatin fragments have been isolated from cells or mononucleosomes and oligonucleosomal arrays were reconstituted from recombinant DNA and purified histone proteins. While chromatin reconstitution using salt dialysis based methods is still central to in vitro chromatin biochemistry, the undefined and mixed nature of PTMs on histones purified from cells has in the past limited the mechanistic investigation of the effects of post-translational marks.

Mechanistically, histone PTMs can act in two non-exclusive ways. On one side, these may directly organize chromatin architecture (i.e. 'compactness' vs 'openness' of chromatin) by modulating internucleosomal contacts [55]. On the other side, the proteins specifically interacting with particular histone PTMs (readers) are thought to mediate downstream functions [6]. The establishment of designer chromatin carrying defined histone PTMs has made major contributions to our understanding of these processes [4-7].

In a landmark study it was shown that a single modification of histone $\mathrm{H} 4$ (H4K16ac) that is implicated in the dosage compensation process in flies impedes chromatin higher order folding (i.e. the transition of primary chromatin fibers into folded structures) when uniformly incorporated into nucleosomal arrays (Figure 3a) [56]. Structural analysis and modeling implied the modulation of a contact of the highly positively charged $\mathrm{H} 4$ tail with an acidic patch within the folded core of $\mathrm{H} 2 \mathrm{~A}$ in this process. Surprisingly, it was reported that a neighboring acetylation mark at H4K20ac had very little effect on array condensation, indicating that only residues in close proximity of $\mathrm{H} 4 \mathrm{~K} 16$ are important for the interaction with H2A [32].

While ubiquitylation of the C-terminus of H2B (H2BK120ub) has been shown to have a similar, overall inhibiting effect on chromatin folding, the mechanism here appears to be distinct and mediated by the addition of the, compared to histones (100-150 aa), large and bulky ubiquitin moiety (76 aa) [57]. Other work also showed that H2BK120ub controls the binding of a large number of factors to chromatin [58].

Compared to H4K16ac, acetylation of a different site within the central, folded part of H3, H3K56ac was shown to have no direct impact on chromatin folding. Here, the effect appears to be on the stability (i.e. dynamics of interaction of histones with DNA) as well as on the enzyme-directed positioning of nucleosomes [59].

Different sites of histone lysine methylation have also been analyzed. For example, it was shown that dimethylation of lysine 79 of histone H3 (H3K79me2) locally alters the nucleosomal surface, whereas trimethylation of lysine 20 of $\mathrm{H} 4$ (H4K20me3) facilitates higher-order chromatin folding [60]. The examples illustrate the complexity of the histone code with the same PTMs on different histone sites having similar effects but also different PTMs on the same histone site having distinct effects.

Besides the analysis of the molecular impact of histone modifications, the chemical biology approaches of designer chromatin have enabled a new level of understanding the binding of proteins to histone marks. A large number of factors specifically recognizing histone PTMs has been identified and characterized on the level of histone peptide-protein domain complexes. These studies have provided fruitful insights into the modes of specific interaction. However, when comparing the set of factors that binds a given histone PTM in the context of a peptide with the set of factors that interacts with such marks in chromatin context on a proteome-wide level, 
Figure 3

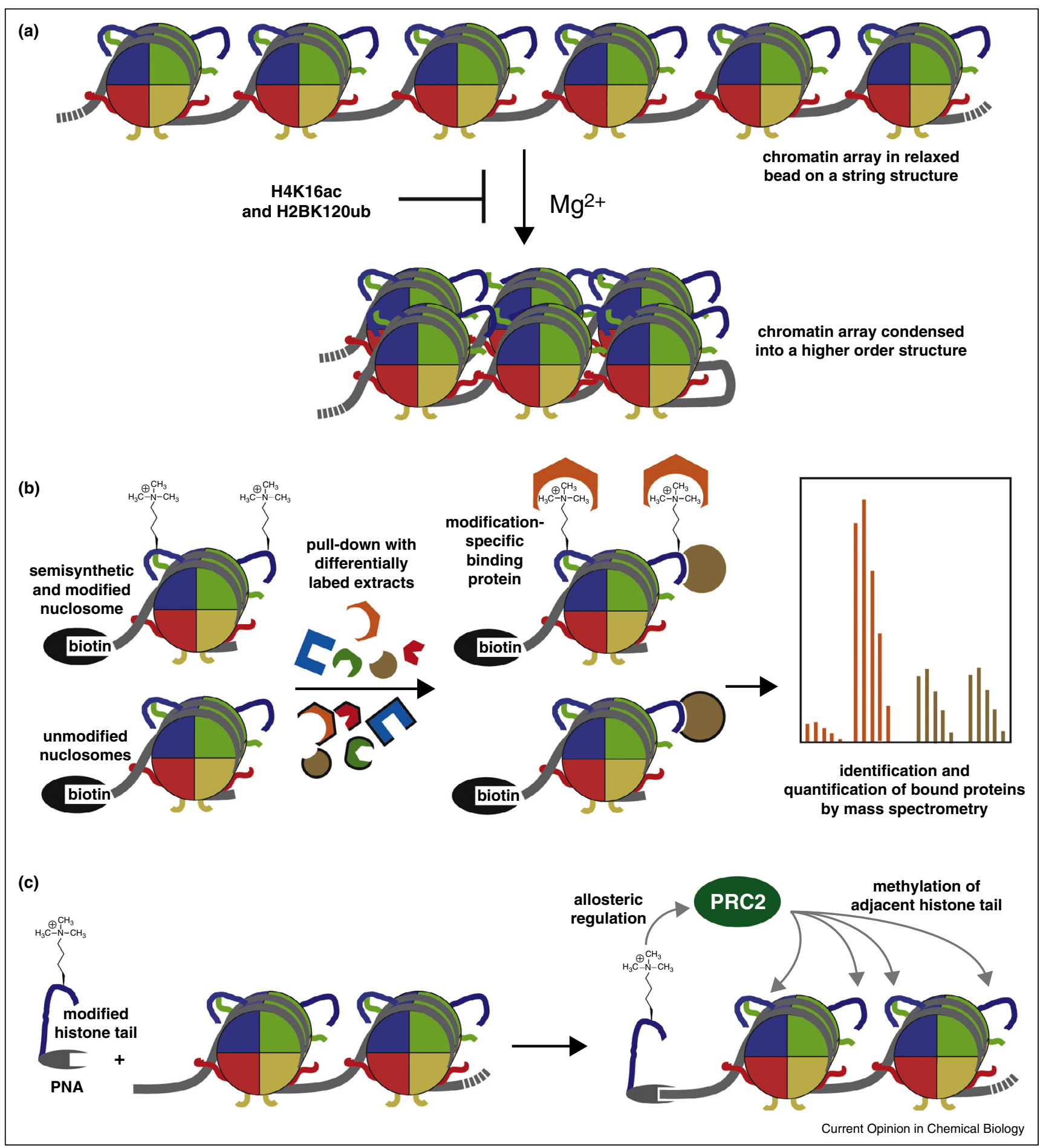

Application of designer chromatin. (a) The folding properties of nucleosome arrays can be analyzed in vitro. Single modifications of histones impair the condensation behavior. (b) Nucleosomes containing histones in distinct modification states can be used as baits for recovering the corresponding reader proteins from cell extracts. Mass spectrometry in combination with specifically labeled cell lysates allows the identification and quantification of nucleosome-bound reader proteins. (c) Synthetic histone tails can be targeted to nucleosomal arrays by PNA-DNA interactions. The modification state of the attached tails can modulate downstream modification of adjacent nucleosomes. 
there is limited overlap (Figure 3b) $\left[61^{\bullet}, 62^{\bullet}, 63\right]$. The results imply that the availability and presentation of histone tails and their modifications is more complex than previously assumed.

Biochemical and structural work has begun to dissect the exact contributions of DNA, other histones and chromatin folding on the interaction properties of defined histone PTMs. For example, it was shown that trimethylation of lysine 9 in $\mathrm{H} 3$ (H3K9me3) directs alternative modes of highly dynamic interaction of heterochromatin protein hHP1 $\beta$ with the nucleosome [64]. Also, the parameters of binding of the PHF1 protein to the trimethylation mark of lysine 36 of H3 (H3K36me3) in nucleosomal context could be worked out. There, it could be shown that this interaction enhances nucleosome accessibility [65].

Finally, designer chromatin templates have enabled the study of the molecular effects and consequences of proteins interacting with histone modifications. For example, for the yeast protein Swi6 a nuclesome bridging mechanism depending on $\mathrm{H} 3 \mathrm{~K} 9 \mathrm{me} 3$ that might be involved in the assembly and spreading of silenced, condensed (i.e. hetero-) chromatin was suggested [66].

\section{Chromatin chemistry is going complex}

While the use of designer chromatin for in vitro chromatin biochemistry is now becoming more and more routine, recent developments are extending the chemical toolbox towards more complex and in vivo chromatin research. For example, conjugates of modified histone tails and peptide nucleic acids (PNAs) for targeted delivery of PTMs were recently described [67]. In order to mediate regulatory functions, histone PTMs need to propagate through larger assemblies of nucleosomes. Prominent examples of this type of 'modification spreading' include the heterochromatin mark $\mathrm{H} 3 \mathrm{~K} 9 \mathrm{me}$, which apparently propagates through recruitment of the heterochromatin binding protein 1 (HP1) and histone methyl-transferase Su(var)3-9 that methylates H3K9 on neighboring nucleosomes. A similar mechanism is considered for $\mathrm{H} 3 \mathrm{~K} 27 \mathrm{me} 3$, which is introduced by the polycomb repressive complex 2 (PRC2) that by itself is regulated by the modification status of H3K27. Histone tail peptides with either PRC2 activating or repressing modification marks were targeted to single nucleosomes or arrays of nucleosomes via conjugated PNAs directed against nearby DNA sequences. The close proximity of the activating and repressing marks were sufficient of either promoting or repressing H3K27 methylation on neighboring nucleosomes although the synthetic tails were only linked to the proximal DNA and not the nucleosomes (Figure 3c) [67].

A further major challenge for deciphering the histone code lies in uncovering the substrate selectivity of chromatin readers, writers and erasers, which need to be probed in high throughput based on the vast number of potential 'histone codons'. The first library of designer nucleosomes representing a large number of combinations of histone PTMs was recently presented [68 $\left.{ }^{\circ}\right]$. In this approach each species of nucleosomes was identified by a unique DNA 'bar code' sequence. After affinity purification, for example via binding to a chromatin factor, next-generation sequencing of the DNA barcodes allowed qualitative and quantitative characterization of the interaction with the distinct modification patterns of the nucleosomes from the library. Modulation of the activity of histone code writers by preexisting modification marks could be analyzed in a similar way by using modification specific antibodies in the pull-down step.

Chromatin of living cells has been largely inaccessible to chemical modification approaches. In two recent studies this 'in vivo barrier' of chromatin chemistry has been breached. Amber suppression technology was used to establish non-natural crosslinking amino acids in chromatin of living cells $\left[69^{\circ \bullet}\right]$. These investigations allowed the dissection of events that initiate chromatin condensation in mitoses mediated by orchestrated writing and erasing of histone marks. Another approach to manipulate chromatin in cells is based on the high affinity of split inteins $\left[70^{\bullet \bullet}\right]$. An intein- $\mathrm{N}$ fragment was expressed as a fusion to core of the histone H2B. The H2B-intein-N construct was efficiently assembled into chromatin in a mammalian cell line despite the size of the intein-N part (102 aa). The complementary intein-C fragment (35 aa) was prepared by solid-phase peptide synthesis together with a short tag sequence for detection by immunoblotting or fluorescence. Intracellular delivery of the intein-C-tag peptide was accomplished by conjugation to a cell-penetratingpeptide (CPP) and specific transfer of the tag sequence to $\mathrm{H} 2 \mathrm{~B}$ by protein trans-splicing was observed, thus opening a new way for direct chemical modification of chromatin. Using the same experimental design, ubiquitin as a PTM of the H2B C-terminal tail could be installed on chromatin. However, this experiment was performed with isolated nuclei, thus circumventing the barrier of the plasma membrane $\left[70^{\circ \bullet}\right]$.

\section{Conclusion}

Protein semisynthesis, amber suppression technology and MLAs have matured from poof-of-concept studies to versatile tools. These experimental strategies are now frequently used in chromatin research. Each method provides advantages and drawbacks in terms of convenience, flexibility (i.e. number and types of modifications) and in vivo applicability.

Installing MLAs and related amino acids mimics into recombinant histones appears straightforward and is widely used. However, the mimics do not cover all chemical properties of the natural modifications and this chemistry is not applicable in living cells. Protein semisynthesis by chemical or chemo-enzymatic means is the 
most flexible approach in terms of number and kinds of modification that can be introduced into a histone protein. However, it cannot always be used in a traceless manner. Also, it is mostly limited to in vitro studies, if not combined with techniques like microinjection for potential cellular applications. Intracellular protein trans-splicing is the only exception reported so far, as it can be applied in vivo when combined with cell penetrating peptides (CPPs) for delivery of the synthetic fragment [54]. Finally, modified amino acids can be efficiently incorporated inside cells without further alteration of the histone sequence using genetic code expansion. However, this approach is limited by the nature of available unnatural amino acids and the remaining difficulty to simultaneously incorporate different PTMs.

While we expect these methods to mature further, chemical control over the modification states of chromatin in living cells is still one of the major challenges in the field. The first steps to introduce chromatin alterations on a global scale are very promising in this regard. However, the selective introduction of defined modification patterns in locally restricted chromatin regions, such as individual promotors, remains a long-term objective. With the focus on new in vivo tools, it appears likely that chemical biology will pave the way for many more discoveries of the rules of the histone code.

\section{Acknowledgments}

We are grateful for financial support by the priority program SPP 1623 of the Deutsche Forschungsgemeinschaft (DFG). We apologize to the authors of the many recent interesting reports in the field of chromatin chemical biology that could not be covered in this review due space constrictions.

\section{References and recommended reading}

Papers of particular interest, published within the period of review, have been highlighted as:

- of special interest

$\bullet$ of outstanding interest

1. Kornberg RD: Structure of chromatin. Ann Rev Biochem 1977, 46:931-954.

2. Jenuwein T, Allis CD: Translating the histone code. Science 2001, 293:1074-1080.

3. Kouzarides T: Chromatin modifications and their function. Cell 2007, 128:693-705.

4. Holt M, Muir T: Application of the protein semisynthesis strategy to the generation of modified chromatin. Annu Rev Biochem 2015, 84:265-290 http://dx.doi.org/10.1146/annurevbiochem-060614-034429.

5. Muller MM, Muir TW: Histones: at the crossroads of peptide and protein chemistry. Chem Rev 2015, 115:2296-2349.

6. Nikolov M, Fischle W: Systematic analysis of histone modification readout. Mol Biosyst 2013, 9:182-194.

7. Schwarzer D: Chemical tools in chromatin research. J Pept Sci 2010, 16:530-537

8. Fischle W: Talk is cheap-cross-talk in establishment, maintenance, and readout of chromatin modifications. Genes Dev 2008, 22:3375-3382.
9. Allis CD, Muir TW: Spreading chromatin into chemical biology Chembiochem 2011, 12:264-279.

10. Fierz B, Muir TW: Chromatin as an expansive canvas for chemical biology. Nat Chem Biol 2012, 8:417-427.

11. Howard CJ, Yu RR, Gardner ML, Shimko JC, Ottesen JJ: Chemical and biological tools for the preparation of modified histone proteins. Top Curr Chem 2015, 363:193-226.

12. Wang ZP, Wang YH, Chu GC, Shi J, Li YM: The study of the chemical synthesis and preparation of histone with posttranslational modifications. Curr Org Synth 2015, 12:150-162.

13. Simon MD, Chu F, Racki LR, de la Cruz CC, Burlingame AL, Panning B, Narlikar GJ, Shokat KM: The site-specific installation of methyl-lysine analogs into recombinant histones. Cell 2007 128:1003-1012.

14. Le DD, Cortesi AT, Myers SA, Burlingame AL, Fujimori DG: Sitespecific and regiospecific installation of methylarginine analogues into recombinant histones and insights into effector protein binding. J Am Chem Soc 2013, 135:2879-2882.

15. Huang R, Holbert MA, Tarrant MK, Curtet S, Colquhoun DR, Dancy BM, Dancy BC, Hwang Y, Tang Y, Meeth K et al.: Sitespecific introduction of an acetyl-lysine mimic into peptides and proteins by cysteine alkylation. J Am Chem Soc 2010, 132:9986-9987.

16. Li F, Allahverdi A, Yang R, Lua GB, Zhang X, Cao Y, Korolev N, Nordenskiold L, Liu CF: A direct method for site-specific protein acetylation. Angew Chem Int Ed Engl 2011, 50:9611-9614.

17. Chalker JM, Lercher L, Rose NR, Schofield CJ, Davis BG: Conversion of cysteine into dehydroalanine enables access to synthetic histones bearing diverse post-translational modifications. Angew Chem Int Ed Engl 2012, 51:1835-1839.

18. Chen Z, Grzybowski AT, Ruthenburg AJ: Traceless semisynthesis of a set of histone 3 species bearing specific lysine methylation marks. Chembiochem 2014, 15:2071-2075.

19. Krishnan S, Collazo E, Ortiz-Tello PA, Trievel RC: Purification and assay protocols for obtaining highly active Jumonji C demethylases. Anal Biochem 2012, 420:48-53.

20. Seeliger D, Soeroes S, Klingberg R, Schwarzer D, Grubmuller H Fischle W: Quantitative assessment of protein interaction with methyl-lysine analogues by hybrid computational and experimental approaches. ACS Chem Biol 2012, 7:150-154.

21. Chatterjee C, McGinty RK, Fierz B, Muir TW: Disulfide-directed histone ubiquitylation reveals plasticity in hDot1L activation Nat Chem Biol 2010, 6:267-269.

22. Wang L, Brock A, Herberich B, Schultz PG: Expanding the genetic code of Escherichia coli. Science 2001, 292:498-500.

23. Gattner MJ, Vrabel M, Carell T: Synthesis of epsilon-Npropionyl-, epsilon-N-butyryl-, and epsilon- $\mathrm{N}$-crotonyl-lysine containing histone $\mathrm{H} 3$ using the pyrrolysine system. Chem Commun (Camb) 2013, 49:379-381.

24. Liu CC, Schultz PG: Adding new chemistries to the genetic code. Annu Rev Biochem 2010, 79:413-444.

25. Li X, Fekner T, Ottesen JJ, Chan MK: A pyrrolysine analogue for site-specific protein ubiquitination. Angew Chem Int Ed Engl $2009,48: 9184-9187$.

26. Sommer S, Weikart ND, Brockmeyer A, Janning P, Mootz HD Expanded click conjugation of recombinant proteins with ubiquitin-like modifiers reveals altered substrate preference of SUMO2-modified Ubc9. Angew Chem Int Ed Engl 2011, 50:9888-9892.

27. Virdee S, Kapadnis PB, Elliott T, Lang K, Madrzak J, Nguyen DP, Riechmann L, Chin JW: Traceless and site-specific ubiquitination of recombinant proteins. J Am Chem Soc 2011, 133:10708-10711.

28. Eger S, Scheffner M, Marx A, Rubini M: Synthesis of defined ubiquitin dimers. J Am Chem Soc 2010, 132:16337-16339.

29. Kim CH, Kang M, Kim HJ, Chatterjee A, Schultz PG: Site-specific incorporation of epsilon- $\mathrm{N}$-crotonyllysine into histones. Angew Chem Int Ed Engl 2012, 51:7246-7249. 
30. Neumann H, Peak-Chew SY, Chin JW: Genetically encoding $\mathrm{N}$ (epsilon)-acetyllysine in recombinant proteins. Nat Chem Biol 2008, 4:232-234.

31. Nguyen DP, Garcia Alai MM, Kapadnis PB, Neumann H, Chin JW: Genetically encoding N(epsilon)-methyl-L-lysine in recombinant histones. J Am Chem Soc 2009, 131:14194-14195.

32. Wilkins BJ, Hahn LE, Heitmuller S, Frauendorf $\mathrm{H}$, Valerius $\mathrm{O}$, Braus GH, Neumann H: Genetically encoding lysine modifications on histone h4. ACS Chem Biol 2015, 10:939-944.

33. Lee S, Oh S, Yang A, Kim J, Soll D, Lee D, Park HS: A facile strategy for selective incorporation of phosphoserine into histones. Angew Chem Int Ed Engl 2013, 52:5771-5775.

34. Park HS, Hohn MJ, Umehara T, Guo LT, Osborne EM, Benner J,

- $\quad$ Noren CJ, Rinehart J, Soll D: Expanding the genetic code of Escherichia coli with phosphoserine. Science 2011 , 333:1151-1154.

First gentically encoded phospho-amino acid

35. Dawson PE, Kent SB: Synthesis of native proteins by chemical ligation. Annu Rev Biochem 2000, 69:923-960.

36. Hackenberger CP, Schwarzer D: Chemoselective ligation and modification strategies for peptides and proteins. Angew Chem Int Ed Engl 2008, 47:10030-10074.

37. Chatterjee C, McGinty RK, Pellois JP, Muir TW: Auxiliarymediated site-specific peptide ubiquitylation. Angew Chem Int Ed Engl 2007, 46:2814-2818.

38. Chiang KP, Jensen MS, McGinty RK, Muir TW: A semisynthetic strategy to generate phosphorylated and acetylated histone H2B. Chembiochem 2009, 10:2182-2187.

39. Fierz B, Kilic S, Hieb AR, Luger K, Muir TW: Stability of nucleosomes containing homogenously ubiquitylated H2A and H2B prepared using semisynthesis. J Am Chem Soc 2012 134:19548-19551.

40. He S, Bauman D, Davis JS, Loyola A, Nishioka K, Gronlund JL, Reinberg D, Meng F, Kelleher N, McCafferty DG: Facile synthesis of site-specifically acetylated and methylated histone proteins: reagents for evaluation of the histone code hypothesis. Proc Natl Acad Sci U S A 2003, 100:12033-12038.

41. McGinty RK, Kim J, Chatterjee C, Roeder RG, Muir TW: Chemically ubiquitylated histone H2B stimulates hDot1Lmediated intranucleosomal methylation. Nature 2008, 453:812-816.

42. Shogren-Knaak MA, Fry CJ, Peterson CL: A native peptide ligation strategy for deciphering nucleosomal histone modifications. J Biol Chem 2003, 278:15744-15748.

43. Siman P, Karthikeyan SV, Nikolov M, Fischle W, Brik A Convergent chemical synthesis of histone H2B protein for the site-specific ubiquitination at Lys34. Angew Chem Int Ed Engl 2013, 52:8059-8063.

44. Ajish Kumar KS, Haj-Yahya M, Olschewski D, Lashuel HA, Brik A

- Highly efficient and chemoselective peptide ubiquitylation. Angew Chem Int Ed Engl 2009, 48:8090-8094.

Method for the generation of a native isopeptide bond of the C-terminus of ubiquitin to a lysine residue.

45. Wan Q, Danishefsky SJ: Free-radical-based, specific desulfurization of cysteine: a powerful advance in the synthesis of polypeptides and glycopolypeptides. Angew Chem Int Ed Engl 2007, 46:9248-9252.

46. Kawakami T, Akai Y, Fujimoto H, Kita C, Aoki Y, Konishi T, Waseda M, Takemura L, Aimoto S: Sequential peptide ligation by combining the Cys-Pro Ester (CPE) and thioester methods and its application to the synthesis of histone $\mathrm{H} 3$ containing a trimethyl lysine residue. Bull Chem Soc Jpn 2013, 86:690-697.

47. Li JB, Li YY, He QQ, Li YM, Li HT, Liu L: One-pot native chemical ligation of peptide hydrazides enables total synthesis of modified histones. Org Biomol Chem 2014, 12:5435-5441.

48. Mootz HD: Split inteins as versatile tools for protein semisynthesis. Chembiochem 2009, 10:2579-2589.
49. Schmohl L, Schwarzer D: Sortase-mediated ligations for the site-specific modification of proteins. Curr Opin Chem Biol 2014, 22:122-128.

50. Shah NH, Muir TW: Split inteins: nature's protein ligases. Isr J Chem 2011, 51:854-861.

51. Popp MW, Ploegh HL: Making and breaking peptide bonds: protein engineering using sortase. Angew Chem Int Ed Engl 2011, 50:5024-5032.

52. Piotukh K, Geltinger B, Heinrich N, Gerth F, Beyermann M, Freund C, Schwarzer D: Directed evolution of sortase A mutants with altered substrate selectivity profiles. J Am Chem Soc 2011, 133:17536-17539.

53. Borra R, Dong D, Elnagar AY, Woldemariam GA, Camarero JA: Incell fluorescence activation and labeling of proteins mediated by FRET-quenched split inteins. J Am Chem Soc 2012, 134:6344-6353.

54. Giriat I, Muir TW: Protein semi-synthesis in living cells. J Am Chem Soc 2003, 125:7180-7181.

55. Winter S, Fischle W: Epigenetic markers and their cross-talk. Essays Biochem Epigenet Dis Behav 2010, 48:45-61.

56. Shogren-Knaak M, Ishii H, Sun JM, Pazin MJ, Davie JR, Peterson CL: Histone H4-K16 acetylation controls chromatin structure and protein interactions. Science 2006, 311:844-847.

57. Fierz B, Chatterjee C, McGinty RK, Bar-Dagan M, Raleigh DP Muir TW: Histone H2B ubiquitylation disrupts local and higherorder chromatin compaction. Nat Chem Biol 2011, 7:113-119.

58. Shema-Yaacoby E, Nikolov M, Haj-Yahya M, Siman P, Allemand E, Yamaguchi Y, Muchardt C, Urlaub H, Brik A, Oren M et al.: Systematic identification of proteins binding to chromatinembedded ubiquitylated H2B reveals recruitment of SWI/SNF to regulate transcription. Cell Rep 2013, 4:601-608.

59. Neumann $\mathrm{H}$, Hancock SM, Buning $\mathrm{R}$, Routh A, Chapman L, Somers J, Owen-Hughes T, van Noort J, Rhodes D, Chin JW: A method for genetically installing site-specific acetylation in recombinant histones defines the effects of H3 K56 acetylation. Mol Cell 2009, 36:153-163.

60. Lu X, Simon MD, Chodaparambil JV, Hansen JC, Shokat KM, Luger K: The effect of H3K79 dimethylation and H4K20 trimethylation on nucleosome and chromatin structure. Nat Struct Mol Biol 2008, 15:1122-1124.

61. Bartke T, Vermeulen M, Xhemalce B, Robson SC, Mann M,

- Kouzarides T: Nucleosome-interacting proteins regulated by DNA and histone methylation. Cell 2010, 143:470-484.

Application of designer chromatin in proteomics.

62. Nikolov M, Stutzer A, Mosch K, Krasauskas A, Soeroes S, Stark H,

- Urlaub H, Fischle W: Chromatin affinity purification and quantitative mass spectrometry defining the interactome of histone modification patterns. Mol Cell Proteom 2011, 10 M110 005371.

See annotation to Ref. $\left[61^{\circ}\right]$

63. Vermeulen M, Eberl HC, Matarese F, Marks H, Denissov S, Butter F, Lee KK, Olsen JV, Hyman AA, Stunnenberg HG et al.: Quantitative interaction proteomics and genome-wide profiling of epigenetic histone marks and their readers. Cell 2010, 142:967-980.

64. Munari F, Soeroes S, Zenn HM, Schomburg A, Kost N, Schroder S, Klingberg R, Rezaei-Ghaleh N, Stutzer A, Gelato KA et al.: Methylation of lysine 9 in histone $\mathrm{H} 3$ directs alternative modes of highly dynamic interaction of heterochromatin protein hHP1beta with the nucleosome. J Biol Chem 2012, 287:33756-33765

65. Musselman CA, Gibson MD, Hartwick EW, North JA, Gatchalian J, Poirier MG, Kutateladze TG: Binding of PHF1 Tudor to H3K36me3 enhances nucleosome accessibility. Nat Commun 2013, 4:2969.

66. Canzio D, Chang EY, Shankar S, Kuchenbecker KM, Simon MD Madhani HD, Narlikar GJ, Al-Sady B: Chromodomain-mediated oligomerization of HP1 suggests a nucleosome-bridging 
mechanism for heterochromatin assembly. Mol Cell 2011, 41:67-81.

67. Brown ZZ, Muller MM, Kong HE, Lewis PW, Muir TW: Targeted histone peptides: insights into the spatial regulation of the methyltransferase PRC2 by using a surrogate of heterotypic chromatin. Angew Chem Int Ed Engl 2015, 54:6457-6461.

68. Nguyen UT, Bittova L, Muller MM, Fierz B, David Y, Houck-

- Loomis B, Feng V, Dann GP, Muir TW: Accelerated chromatin biochemistry using DNA-barcoded nucleosome libraries. Nat Methods 2014, 11:834-840.
First library of designer nuclosomes for high-troughput chromatin research.

69. Wilkins BJ, Rall NA, Ostwal Y, Kruitwagen T, Hiragami-Hamada K,

- Winkler M, Barral Y, Fischle W, Neumann H: A cascade of histone modifications induces chromatin condensation in mitosis. Science 2014, 343:77-80.

One of so far only two examples of in vivo chromatin chemistry.

70. David Y, Vila-Perello M, Verma S, Muir TW: Chemical tagging

-. and customizing of cellular chromatin states using ultrafast trans-splicing inteins. Nat Chem 2015, 7:394-402.

See annotation to Ref. [69*日]. 